\title{
INEQUALITIES FROM LORENTZ-FINSLER NORMS
}

\section{Nicuşor Minculete*, Christian Pfeifer and Nicoleta Voicu}

\begin{abstract}
We show that Lorentz-Finsler geometry offers a powerful tool in obtaining inequalities. With this aim, we first point out that a series of famous inequalities such as: the (weighted) arithmetic-geometric mean inequality, Aczél's, Popoviciu's and Bellman's inequalities, are all particular cases of a reverse Cauchy-Schwarz, respectively, of a reverse triangle inequality holding in Lorentz-Finsler geometry. Then, we use the same method to prove some completely new inequalities, including two refinements of Aczél's inequality.
\end{abstract}

Mathematics subject classification (2010): 26D15, 46C05, 53B40, 53C50.

Keywords and phrases: Cauchy-Schwarz inequality, Lorentz-Finsler norms.

\section{REFERENCES}

[1] A. B. Aazami And M. A. Javaloyes, Penrose singularity theorem in a Finsler spacetime, Class. Quant. Grav. 33 (2016) no. 2, 025003, doi:10.1088/0264-9381/33/2/025003.

[2] J. ACZÉL, Some general methods in the theory of functional equations in one variable, New applications of functional equations (Russian), Uspehi Mat. Nauk (N. S.), 11, 69 (3) (1956), 3-68.

[3] G. S. Asanov, Finsler space with an algebraic metric defined by a field of frames, J. Math. Sci. 13 (1980), 588-600.

[4] D. BAo, S. S. Chern And Z. Shen, An Introduction to Finsler-Riemann Geometry, Springer, New York, 2000.

[5] R. Bellman, On an inequality concerning an indefinite form, Amer. Math. Monthly 63 (1956), 108109.

[6] J. K. Beem, P. E. Ehrlich, And K. L. Easley, Global Lorentzian geometry, volume 202 of Monographs and Textbooks in Pure and Applied Mathematics. Marcel Dekker, Inc., New York, second edition, 1996.

[7] A. Bernal, M. A. Javaloyes And M. SÁnchez, Foundations of Finsler spacetimes from the Observers' Viewpoint, Universe 6 (2020) no. 4, 55, doi:10.3390/universe6040055.

[8] N. BRîNZEI, Projective relations for $m$-th root metric spaces, Journal of the Calcutta Mathematical Society 5 (1-2), (2009), 21-35.

[9] E. Caponio, G. Stancarone, On Finsler spacetimes with a timelike Killing vector field, Class. Quant. Grav., 35 (2018) 8, 085007.

[10] E. CAPONio, M. A. JaVAloyes And M. SÁncheZ, Wind Finslerian structures: from Zermelo's navigation to the causality of spacetimes, [arXiv:1407.5494 [math.DG]].

[11] M. Hohmann, C. Pfeifer, N. Voicu, Cosmological Finsler spacetimes, Universe 6 (5), 65 (2020).

[12] M. Hohmann, C. Pfeifer AND N. VoicU, Finsler gravity action from variational completion, Phys. Rev. D 100, no. 6 (2019), 064035, doi:10.1103/PhysRevD.100.064035.

[13] M. A. Javaloyes, M. SÁNCHeZ, On the definition and examples of cones and Finsler spacetimes, Rev. R. Acad. Cienc. Exactas Fis. Nat. Ser. A Mat., RACSAM 114, 30 (2020).

[14] M. A. Javaloyes, M. SÁnchez, On the definition and examples of Finsler metrics, Ann. Sc. Norm. Super. Pisa Cl. Sci. 5, XIII, (2014), 813-858.

[15] M. A. JaVAloyes AND H. Vitorio, Some properties of Zermelo navigation in pseudo-Finsler metrics under an arbitrary wind, Houston J. Math., 44, (4) (2018), 1147-1179.

[16] V. K. KRopina, On projective two-dimensional Finsler spaces with a special metric, Trudy Sem. Vektor. Tenzor. Anal., 11 (1961), 277-292. 
[17] C. Lammerzahl, V. Perlick And W. Hasse, Observable effects in a class of spherically symmetric static Finsler spacetimes, Phys. Rev. D 86, 104042 (2012), doi:10.1103/PhysRevD.86.104042.

[18] N. Minculete, R. PĂLtĂneA, Improved estimates for the triangle inequality, J. Ineq. Appl. (2017)(1), 1-12.

[19] E. MinguZzI, Lorentzian causality theory, Living Rev. Relativ. 22: 3 (2019).

[20] E. Minguzzi, Light Cones in Finsler Spacetime, Comm. Math. Phys., 334 (2015), 1529-1551.

[21] E. MinguZzI, Convex neighborhoods for Lipschitz connections and sprays, Mon. Math. 177 (2015), 569-625, doi:10.1007/s00605-014-0699-y.

[22] D. S. Mitrinović, Analytic Inequalities, Springer, Berlin-Heidelberg-New York, 1970.

[23] D. S. Mitrinović, J. PeČarić, A. M. Fink, Classical and New Inequalities in Analysis, Springer, Dordrecht 1993.

[24] B. O’NeILl, Semi-Riemannian Geometry With Applications to Relativity, Academic Press, San Diego-London, 1983.

[25] V. Perlick, Ray Optics, Fermat's Principle, and Applications to General Relativity, No. 61 in Lecture Notes in Physics, Springer, (2000).

[26] C. Pfeifer AND M. N. Wohlfarth, Causal structure and electrodynamics on Finsler spacetimes, Phys. Rev. D 84 (2011), 044039, doi:10.1103/PhysRevD.84.044039.

[27] C. PFEIFER, The Finsler spacetime framework: backgrounds for physics beyond metric geometry, DESY-THESIS-2013-049.

[28] C. Pfeifer And D. Siemssen, Electromagnetic potential in pre-metric electrodynamics: Causal structure, propagators and quantization, Phys. Rev. D 93 (2016) no. 10, 105046, doi:10.1103/PhysRevD.93.105046.

[29] C. Pfeifer, Finsler spacetime geometry in Physics, Int. J. Geom. Meth. Mod. Phys. 16, no. supp02, 1941004, (2019), doi:10.1142/S0219887819410044.

[30] T. Popoviciu, On an inequality (Romanian), Gaz. Mat. Fiz. Ser. A 8(64) (1959), 451-461.

[31] R. Punzi, M. N. Wohlfarth And F. P. Schuller, Propagation of light in area metric backgrounds, Class. Quant. Grav. 26 (2009), 035024, doi:10.1088/0264-9381/26/3/035024.

[32] G. Randers, On an Asymmetrical Metric in the Four-Space of General Relativity, Phys. Rev. 59 (1941), 195-199, doi:10.1103/PhysRev.59.195.

[33] D. Raetzel, S. Rivera And F. P. Schuller, Geometry of physical dispersion relations, Phys. Rev. D 83 (2011), 044047, doi:10.1103/PhysRevD.83.044047. 